\title{
Factors Affecting Liver Regeneration in Donors' Grafts in Living Donor Liver Transplant (LDLT) a Center Experience
}

\author{
Morsi Mohamed ${ }^{1, ~ *}$, Wael Mansy ${ }^{1}$, Osama Khalil ${ }^{2}$, Yehia Attwa ${ }^{1}$, Magdy Amin ${ }^{3}$, Karim Boudjema $^{4}$ \\ ${ }^{1}$ Hepato-Pancreatico-Biliary Unit, General Surgery Department, Zagazig University, Zagazig, Egypt \\ ${ }^{2}$ General Surgery Department, Zagazig University, Zagazig, Egypt \\ ${ }^{3}$ Liver Transplant Unit in Hepato-Biliary Department, El-Maadi Armed Forces Hospital, Cairo, Egypt \\ ${ }^{4}$ Hepato-Biliary Surgery and Liver Transplant Unit, Rennes University, Rennes, France \\ Email address: \\ drmorsi@yahoo.com (M. Mohamed), drwaelmansy@hotmail.com (W. Mansy), osama100khalil@yahoo.com (O. Hamed Khalil), \\ yzakaria1956@gmail.com (Y. Attwa), magdyamin@gmail.com (M. Amin), karim.boudjema@ chu-rennes.fr (K. Boudjema) \\ ${ }^{*}$ Corresponding author
}

\section{To cite this article:}

Morsi Mohamed, Wael Mansy, Osama Khalil, Yehia Attwa, Magdy Amin, Karim Boudjema. Factors Affecting Liver Regeneration in Donors' Grafts in Living Donor Liver Transplant (LDLT) a Center Experience. Journal of Surgery. Vol. 7, No. 4, 2019, pp. 87-95. doi: $10.11648 /$ j.js.20190704.11

Received: May 14, 2019; Accepted: June 23, 2019; Published: July 2, 2019

\begin{abstract}
Background: Liver regeneration for donors' graft in living donor liver transplantation (LDLT), is an example for liver regeneration in normal liver. In this study we spotted the light on liver regeneration in donors after LDLT, focusing on factors affecting it. Aim: To study the impact of different factors on liver regeneration in donors of living donor liver transplantation. Patients and methods: Between May 2013 and June 2019, 80 living donor liver transplants were performed in the liver transplant unit in EL-Maadi Armed Forces Hospital. All donors who provided liver grafts underwent volumetric spiral computed tomography (CT) scans preoperatively and postoperatively at time intervals of 1 week and 1, 3 and 6 months. Patients' demographics, surgical data, and postoperative outcomes were correlated with liver regeneration data. 63 patients were males and 17 patients were females, with mean age $27.97 \mathrm{yrs} \pm 5.3$. They provided $\{72\}$ right lobe (RL) grafts and 8 right posterior sector (RP) grafts. Results: No donor operation was aborted and surgical morbidity rate was $\{33.75 \%\}$, mostly biliary. All RP donors achieved complete liver regeneration after 3 months; only four RL donors achieved complete liver regeneration at 6 months. Conclusion: In this study, it was found that there were factors affecting regeneration like age, gender of the patient and graft size. But no effect was found with many factors as peri-operative liver function tests, steatosis, BMI, operative time, blood loss, blood transfusion and postoperative complications.
\end{abstract}

Keywords: Liver Transplantation, Liver Regeneration, Living Donors

\section{Introduction}

Liver transplantation is one of the most effective ways of treatment for many patients with liver failure whatever the cause. It gives a strong impact on the patient outcome [1]. Over the past 15 years LDLT had been used and developed tremendously [2]. Liver regeneration is critical in adult living donor liver transplantation (LDLT), and size considerations affect the selection of appropriate donor and recipient pairs [3]. Single center studies have shown that recipients have rapid liver regeneration but that many donors do not regain total liver volume, even after 1 year [4].
Normal Liver regeneration is a complicated process as it depends on the activation of more than one hundred genes and involvement of numerous growth factors [5]. Portal hemodynamics, vascular outflow, graft to recipient weight ratios (GRWR), humoral factors, and graft quality have all been implicated in affecting liver regeneration. Left lobe donors provide even smaller grafts, and makes the procedure potentially safer for the donor but increases the risk for the recipient $[6,7]$.

This process is highly effective, after hemi-hepatectomy and liver donation; the volume and function of the liver are largely restored after two or three weeks, and completely after six months [8]. We believe that if the liver quality and the 
regeneration rate are good then donor safety can be ensured by preserving approximately one-third of the total liver volume [9]. Few studies concerned with liver regeneration in healthy living donors. But many studies discussed liver regeneration after partial hepatectomy for benign and malignant tumors [10, $11]$.

\section{Patients and Methods}

Between May 2013 and June 2019, the surgical team in liver transplant unit in El-Maadi armed forces compound hospital performed 80 LDLT using right lobe grafts from 72 healthy donors and right posterior sector grafts (segment VI\&VII) from 8 healthy donors. Twice weekly, a multidisciplinary screening committee composed of the members of the surgical team, radiologist and internal medicine doctors met to review the recipient donor couple and evaluate the recipient risk, indication of transplant and other options if transplant was not indicated. The most common indications were liver cirrhosis 44 patients $(55 \%)$ and HCC 20 cases $(25 \%)$ caused by HCV infection, while other indications found in 16 patients $(20 \%)$.

Our donors' criteria included; age (18-45yrs), should related to recipient and blood group compatibility. All the donor demographics were recorded regarding age, sex, weight and height. BMI, blood group, degree of steatosis, type of the graft, CT total liver volume, and CT right graft volume were evaluated. Potential donors are told of the risk of donor and morbidity and mortality.

All donors underwent liver function tests preoperatively and daily at the $1^{\text {st }}$ week postoperative, then at 1,3 and 6 months postoperatively. All donors underwent liver biopsy to exclude donors with macro-vesecular steatosis more than $10 \%$. Liver US Duplex was done to assess the hepatic venous and portal anatomy preoperatively then at day 1, 3, 7 postoperatively to exclude portal vein thrombosis or fluid collection. It also was done postoperatively at 1, 3 and 6 months, to detect the increase in the portal flow and presence or absence of portal hypertension.

Also Multiphase abdominal computed tomography angiography (CTA) was performed preoperatively to delineate liver morphology, volume, and vascular anatomy. Then, it was performed at 1 week, 1, 3 and 6 months postoperatively to detect increasing ratio of regeneration. The CT volumetry protocol used to assess donor liver volume; was using manual measurements to calculate liver volumes by hand-tracing the liver outline on the axial portal venous phase images (Figure 1).
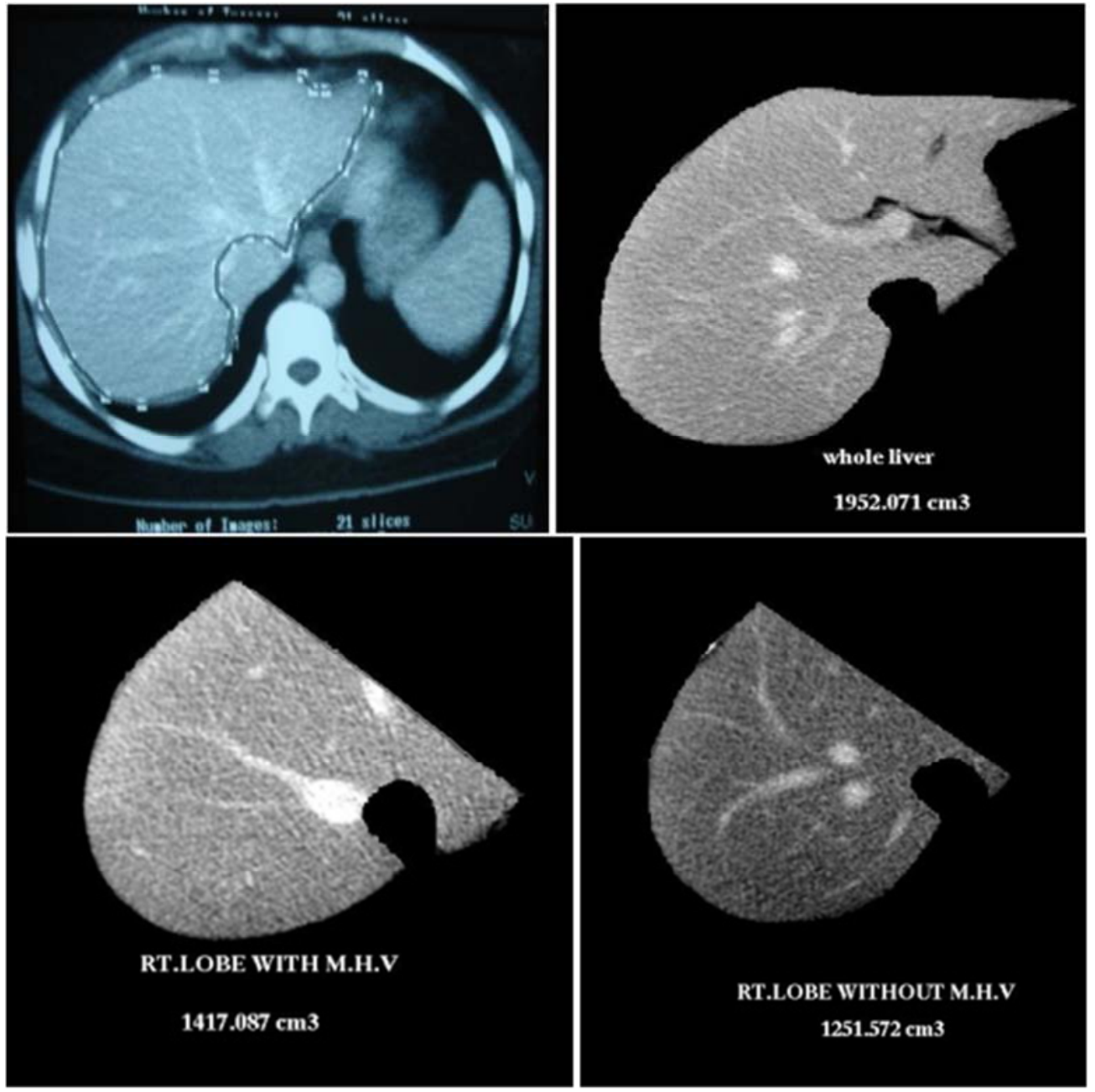

Figure 1. CT Volumetry Protocol. A. Abdominal CT outlines liver for volumetry. B. Whole live volume. C. Right lobe with MHV. D. Right lobe without MHV. 
On preoperative CT scans using hepatic veins as guidance, we measured the total liver volume; the volume of the right lobe of the liver; the remnant liver volume, which included the (caudate lobe, IV, bi-segment II \& III). For the right lobe, a line parallel to the right side of the middle hepatic vein was drawn, and the circumference of the right side of the liver was marked manually in slices $1 \mathrm{~cm}$ thick. When the estimated liver remnant volume is less than $35 \%$, the candidate, was considered unsuitable as a living donor.

The graft recipient weight ratio (GRWR) had been used to assess the graft size of a potential donor, and values of less than $0.8 \%$ had been associated with increased posttransplantation mortality and morbidity.

Seventy two donors underwent right hepatectomy; the remained liver was the left lobe with preservation of the MHV in the donors. While in the eight donors of right posterior sector graft, we took segment VI, VII with preservation of left lobe plus segment V, VIII in the donors.

All the operative details were recorded regarding the length of the operation, blood loss, autologous blood and fresh frozen plasma (FFP) transfusion, actual right lobe graft weight, and posterior sector graft weight and donor remnant volume. All donor complications, defined as any unexpected events, were collected prospectively and recorded, both minor and major complications were recorded. All donors were monitored for 6 months postoperatively for surgical and medical complications.

Statistical Analysis

Donor remnant volume $\{\mathrm{DRV}\}$ was calculated as the estimated total liver volume $\{\mathrm{TLV}\}$ minus the actual graft weight $\{\mathrm{AGW}$. Regeneration was expressed as a percent of the original TLV using follow-up computed tomography $\{\mathrm{CT}\}$ liver volume. Total liver volume by $\mathrm{cm}^{3}$ was recorded at the time interval of follow-up.

Increased ratio was calculated by this formula \{regeneration LV - DRV $/ \mathrm{DRV}^{*} 100$ \}. As regeneration LV was the new remnant liver volume at the time interval of follow-up and DRV was the remnant liver volume (the donor remnant volume at day 0). Data are expressed as mean + standard deviation. Person's correlation was used to analyze associations between two continuous variables. Statistical analyses were done using the statistical package for the social science $\{$ SPSS $\}$. A $p$ value $<0.05$ was considered statistically significant.

\section{Results}

Eighty living donors with compatible blood group s were evaluated, seventy two (56 males and 16 females) underwent right hepatic resection with preservation of $\mathrm{MHV}$ in the donor while only eight ( 7 males and 1 female) underwent right posterior sector (segment VI, VII) hepatic resection. Most common blood group was O (38.75\%). Regarding age donors were relatively young $(27.97 \pm 5.3$ years $)$ with normal body weight and height (Body mass index 25.2 \pm 3.37 ) (table 1).

Table 1. Donor's characteristics.

\begin{tabular}{lll}
\hline Variants & & Number of donors \\
\hline Sex: & Male & 63 \\
& Female & 17 \\
AGE: & 10-20yrs & $27.97 \pm 5.3$ \\
& $21-30 \mathrm{yrs}$ & 6 \\
& $31-40 \mathrm{yrs}$ & 48 \\
BMI: & & 26 \\
BLOOD GROUP : & & $25.2 \pm 3.37$ \\
& A & \\
& B & $25\{20+\mathrm{ve} \& 5$-ve $\}$ \\
& O & $15\{13+\mathrm{ve} \&$ 2-ve $\}$ \\
& AB & $31\{27+\mathrm{ve} \&$ 4-ve $\}$ \\
Liver biopsy: & & $98+\mathrm{ve} \& 1$-ve $\}$ \\
& No steatosis & 52 \\
& Steatosis $<5 \%$ & 18 \\
& Steatosis 5- 10\% & 10 \\
Type of the graft: & & 72 \\
& Right lobe & 8 \\
\hline
\end{tabular}

The donor operative time was $(6.16 \pm 0.88)$ hours with an estimated blood loss $(1118 \pm 833 \mathrm{ml}$.). Blood transfusion was in 14 donors with $(2.67 \pm 2.03)$ units and fresh frozen plasma in 15 donors with $(2.7 \pm 1.38$ units). ICU length stay for all donors was $(2 \pm 1)$ days and hospital stay for all donors was $(9 \pm 3)$ days (table 2).

Table 2. Donors' operative data.

\begin{tabular}{lllll}
\hline Variants & Min & Max & Mean & SD \\
\hline operation time (Hour) & 4 & 8 & 6.16 & \pm 0.88 \\
Blood loss (ml) & 300 & 4500 & 1118 & \pm 833 \\
Blood transfusion (unit) & 1 & 8 & 2.67 & \pm 2.03 \\
FFP transfusion (unit) & 1 & 11 & 2.7 & \pm 1.38 \\
Hospital stay (day) & 7 & 22 & 9.3 & \pm 3.32 \\
\hline
\end{tabular}

The preoperative liver volume measured by CT volumetry program was $\left(1677 \pm 225.3 \mathrm{~cm}^{3}\right)$ for right lobe graft and $\left(1653.6 \pm 187.1 \mathrm{~cm}^{3}\right)$ for right posterior sector graft. Multidetector CT scan was excellent in predicting mean right lobe liver volume \{predicted $957.8 \pm 177.1 \mathrm{ml}$ vs. actual $858.9 \pm$ $165.3 \mathrm{~g}\} \mathrm{P}<0.001$ by paired $\mathrm{t}$ test. The actual mean right

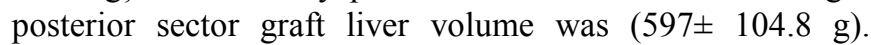
Actual graft volume was $89.7 \%$ of the CT predicted right lobe liver volume. The donor's remnant liver volume was $(47.1 \pm 5.2 \%)$ of TLV in right lobe and $(63.7 \pm 4.8 \%)$ of TLV in right posterior. The mean GRWR was $1.05 \%$ (table 3 ). 
Table 3. Donor liver graft characteristics.

\begin{tabular}{|c|c|c|c|}
\hline Variants & Min & Max & Mean \\
\hline CT total liver volume $\left(\mathrm{cm}^{3}\right)$ (right lobe graft) & 1239 & 2200 & $1677 \pm 225.3$ \\
\hline CT total liver volume $\left(\mathrm{cm}^{3}\right)$ (right posterior graft) & 1451.6 & 1841.73 & $1653.6 \pm 187.1$ \\
\hline CT right lobe liver volume $\left(\mathrm{cm}^{3)}\right.$ & 650 & 1360 & $957.8 \pm 177.1$ \\
\hline Actual right lobe graft weight (gm) & 510 & 1166 & $858.9 \pm 165.3$ \\
\hline Donors of RT lobe graft remained volume (\%) & 37.1 & 60 & $47.1 \pm 5.2$ \\
\hline Actual right posterior sector graft weight (gm) & 510 & 726 & $597 \pm 104.8$ \\
\hline
\end{tabular}

\subsection{Liver Regeneration and Function Recovery}

Liver regeneration was measured at 1 week and at 1, 3, 6 months postoperatively and calculated using helical CT scan. The eight donors of the right posterior graft nearly reached the preoperative whole liver volume after 3 months. Four donors of right lobe graft (one male, one female) also, nearly reached the preoperative whole liver volume after 6 months. Postoperative liver enzymes revealed initial high elevation in the first 3 days then declined gradually, reached the baseline after 7 days postoperative. The total bilirubin returned to normal level at post-operative day pod\#7 except in 4 donors ( 2 of them complicated with cholangitis and the other two complicated with intra-operative bleeding and blood transfusion) returned to normal level at pod\#30. In all right lobe donors we didn't harvest the MHV to prevent outflow obstruction of segment IV and to maintain regeneration of the caudate lobe and segment IV.
Duplex US was done at first two days to exclude any portal vein thrombosis and fluid collection and then at the time of CT volumetry. The liver duplex revealed increased in the portal flow and to some extent increased portal pressure leading to transient splenomegaly for 3 months then return to normal size afterwards.

\subsection{Factors Affecting Liver Regeneration}

\subsubsection{Graft Type}

Donor residual liver volume was $(63.7 \pm 4.8 \%)$ of RT posterior graft reached to $(99.6 \pm 0.21 \%)$ at POD\# 90 , and was $(47.1 \pm 5.4 \%)$ of RL donors reached to $(95.2 \pm 1.7 \%)$ at POD\# 180. The increased ratios of the remnant liver were high in the RL donors when compared with the RT Posterior donors $\{\mathrm{P}<0.001\}$. However, the liver enzymes and total bilirubin levels were high in the RL donors in the 1 st week more than RT post. Graft (tables 4, 5) (Figure 2).

Table 4. Donor morbidity.

\begin{tabular}{ll}
\hline Complications & Number of patients \& management \\
\hline Biloma & 6 (5 of 6 need US pigtail drainage) \\
Biliary leak & 1 (one had ERCP) \\
Postoperative bleeding & 1 (reoperation done for him) \\
Postoperative hematoma & 3 (1 of 3 need reoperation) \\
Postoperative fluid collection & 2 (US aspiration done for both) \\
Pneumonia & 2 (medical) \\
Cholangitis & 2 (medical) \\
Wound problem & 6 (4 seroma, 1 wound infection \& \\
Transient ascites & 1 with stitch sinus who reoperated) \\
Scrotal edema & 7 (medical) \\
Pleural effusion & 1 (medical) \\
Paralytic ileus & 7 (1 of 7 need thoracocentesis) \\
HAV infection & 3 (medical) \\
\hline
\end{tabular}

Table 5. Liver graft regeneration, according to the type of the graft.

\begin{tabular}{|c|c|c|c|c|c|c|}
\hline Days & Graft Type & $\begin{array}{l}\text { Remained Liver Vol. } \\
\left(\mathrm{cm}^{3}\right)\end{array}$ & $\begin{array}{l}\% \text { Of The Original } \\
\text { Liver Vol. }\end{array}$ & $\begin{array}{l}\% \text { Of Regeneration Per } \\
\text { Day From Previous Visit }\end{array}$ & $\begin{array}{l}\text { Increased Ratio Of } \\
\text { Remnant Vol. }\end{array}$ & $P$ Value \\
\hline \multirow{2}{*}{0} & RT post.: 8 & $1047 \pm 130.2$ & $63.7 \pm 4.8$ & & & \\
\hline & RT lobe: 72 & $793.7 \pm 146.7$ & $47.1 \pm 5.4$ & & & \\
\hline \multirow{2}{*}{7} & RT post.: 8 & $1431.9 \pm 115$ & $86.7 \pm 1.6$ & $3.28 \pm 0.23$ & $37.4 \pm 11.3$ & $<0.001$ \\
\hline & RT lobe: 72 & $1170.8 \pm 169.4$ & $69.8 \pm 5.1$ & $3.24 \pm 0.73$ & $49.5 \pm 12$ & $<0.001$ \\
\hline \multirow{2}{*}{30} & RT post.: 8 & $1582 \pm 129.9$ & $95.1 \pm 1$ & $0.36 \pm 0.044$ & $51.1 \pm 12$ & $<0.001$ \\
\hline & RT lobe: 72 & $1360.3 \pm 186.7$ & $80.6 \pm 1.9$ & $0.47 \pm 0.08$ & $71.4 \pm 23.5$ & $<0.001$ \\
\hline \multirow{2}{*}{90} & RT post.: 8 & $1647 \pm 148.9$ & $99.6 \pm 0.21$ & $0.075 \pm 0.003$ & $57.3 \pm 14.2$ & $<0.001$ \\
\hline & RT lobe: 72 & $1488.1 \pm 202$ & $88.5 \pm 2$ & $0.13 \pm 0.03$ & $87.5 \pm 25.4$ & $<0.001$ \\
\hline 180 & RT lobe: 72 & $1599.8 \pm 214$ & $95.2 \pm 1.7$ & $0.075 \pm 0.019$ & $101.6 \pm 26.9$ & $<0.001$ \\
\hline
\end{tabular}




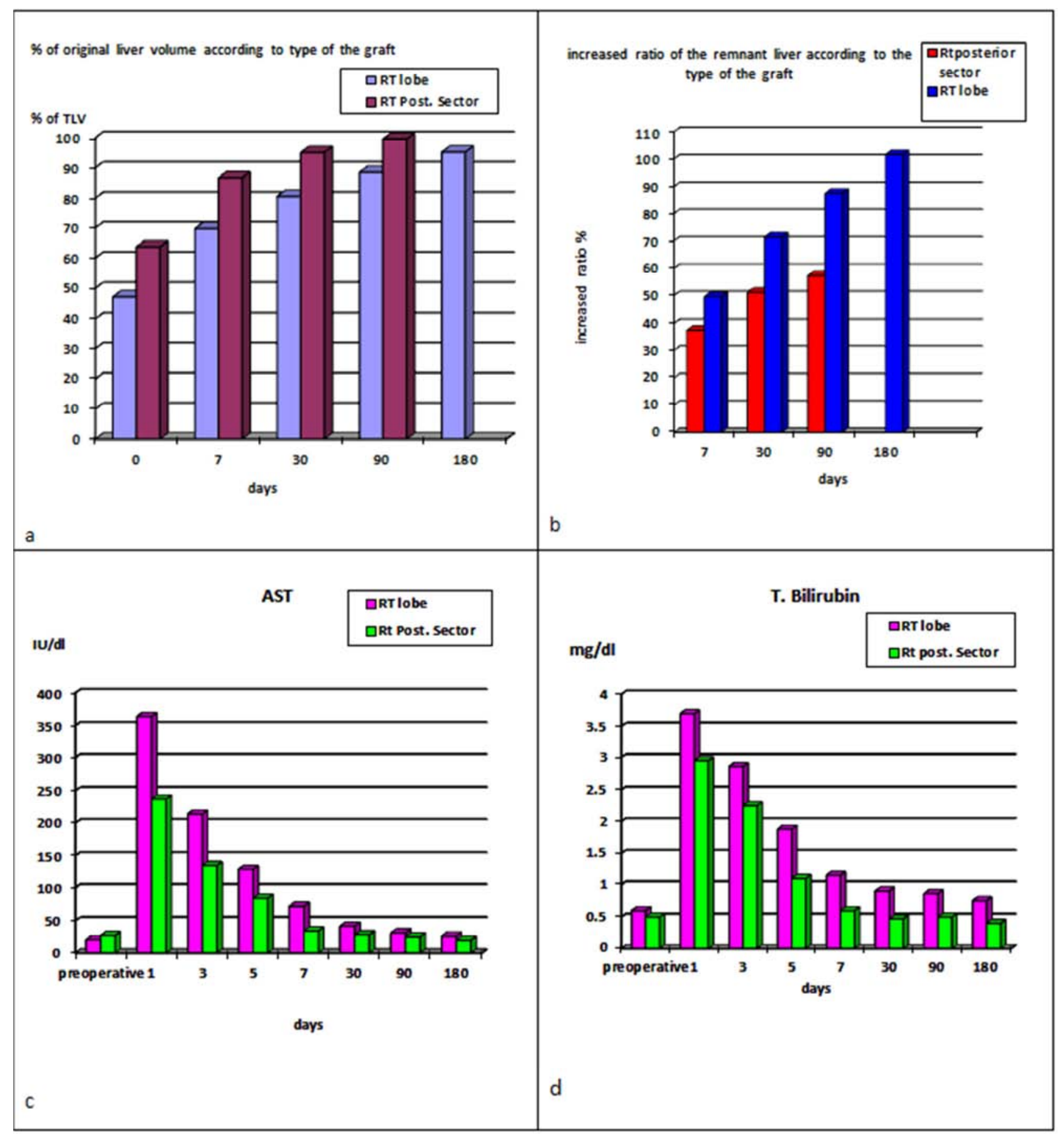

Figure 2. Postoperative liver regeneration and function recovery according to the type of the graft. A:\% of TLVs. B: increased ratio of the remnant liver volume. C: AST. D: T. Bilirubin.

\subsubsection{Donor Sex}

Right hepatectomy was carried in 16 female donors and 56 male donors. Donor residual liver volume was $(47.9 \pm 5.3 \%)$ in male donors, reached to $(95.3 \pm 1.8 \%)$ at POD \#180, and was $(43.6 \pm 4.7 \%)$ in female donors, reached to $(94.6 \pm$ $1.1 \%$ ) at POD $\# 180$. The remnant liver of female group showed rapid regeneration than male group especially in POD\#7, increased ratios were $(68.8 \%)$ in females vs.(44.2\%) in males and remnant liver in female group increased from $(43.6 \%)$ to $(74.3 \%)$ of TLV vs. from $(47.9 \%)$ to $(68.7 \%)$ of
TLV in male group , ( $\%$ of regeneration per day) was $(4.39 \%)$ in female group vs. $(2.97 \%)$ in male group in POD $\# 7\{\mathrm{P}<0.001\}$.

\subsubsection{Donor Age}

All RL donors were analyzed. There were six donors below 20 years (group 1), forty eight donors between 20 and 30 years (group 2), and twenty six donors above 30 years (group 3). The preoperative TLV, remnant liver volume showed no significant difference between age group s. The liver regeneration was slow in the teenagers at POD \#7, the 
increased ratio was $(33.9 \%)$ in group $1,(46.9 \%)$ in group 2 and $(53.9 \%)$ in group $3(\mathrm{P}<0.001)$.

\subsubsection{Remained Liver/Donor Weight $(R L / D W)$ Ratio}

Donors divided to group s according to RL/DW ratio into group 1 (between 0.6 and $0.8, \mathrm{~N}=18$ ), group 2 (between 0.81 and 1, N=29) and group 3 (between 1.01 and 1.2, $\mathrm{N}=33$ ). The remnant liver volume was $(41.2 \%)$ of TLV increased to $(95.8 \%)$ of TLV in group $1,(47.7 \%)$ of TLV increased to (95\%) of TLV in group 2 and $(54.3 \%)$ of TLV increased to $(95.1 \%)$ of TLV in group 3 . The increased ratios were high in group $1(\mathrm{P}<0.001)$.

\subsubsection{Remnant Liver Size}

We divided all donors to group $\mathrm{s}$ according to remained liver volume to (group 1, N=6) less than $40 \%$ of preoperative TLV, (group 2, $\mathrm{N}=56$ ) between $40 \%$ and $50 \%$ and (group 3, $\mathrm{N}=18)$ more than $50 \%$. The remnant liver volume was $(69.1 \%)$ of TLV increased to $(94.1 \%)$ of TLV in group 1 , $(69.1 \%)$ of TLV increased to $(95.1 \%)$ of TLV in group 2 and $(69.5 \%)$ of TLV increased to $(94.6 \%)$ of TLV in group 3 . The increased ratios were high in group $1(\mathrm{P}<0.001)$ (Figure 3$)$.

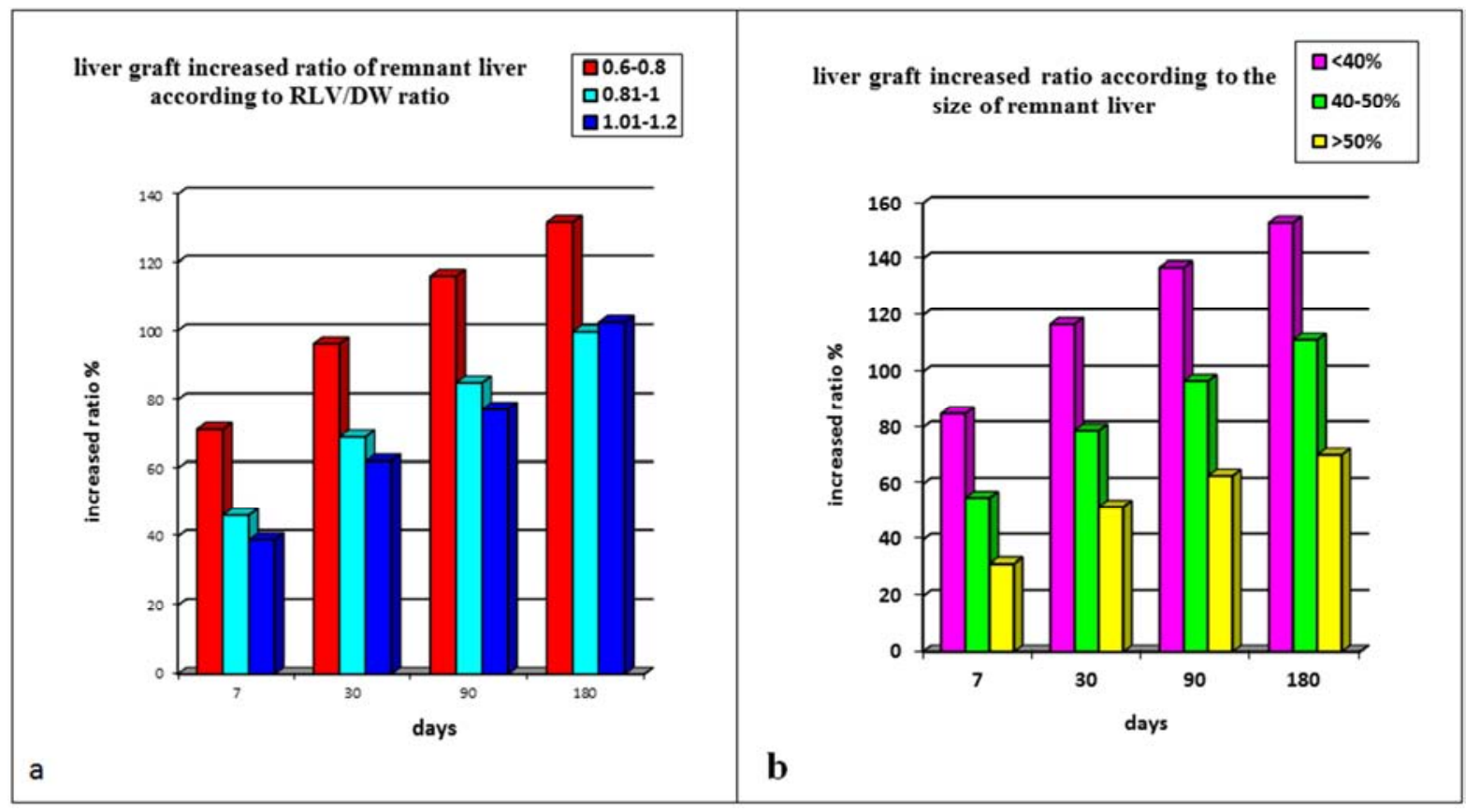

Figure 3. Postoperative liver regeneration. A: according to $R L / D W$ ratio. B: according to size of the remnant liver.

\subsubsection{Fatty Change of Donor Liver}

Donors divided into 3 group s, group 1 (no steatosis) as no fatty degeneration in 52 donors, group 2 with fatty change less than $5 \%$ in 18 donors and group 3 with fatty change between 5 and $10 \%$ in 10 donors. There was no significant difference between group s on regenerated liver volume.

\subsubsection{Postoperative Complications and Blood Transfusion}

Donors divided according to blood transfusion into 2 group s, no blood transfusion in group 1 in 23 donors and with blood transfusion in group 2 in 57 donors. Also we divided the donors according to postoperative complications into group 1 (morbidity) in 27 donors and group 2 (no morbidity) in 53 donors. There was no significant difference between group $\mathrm{s}$ on regenerated liver volume.

\section{Discussion}

Liver transplantation is a recent solution for patients with end stage liver disease. Graft of the right lobe of the liver was world wild trend for living donor liver transplantation because of enough volume and function of right lobe to meet the metabolic need of adult recipient [8]. The development of refinements in surgical techniques, unique anatomy and physiology of the liver expands living donor partial liver transplantation [12]. Appropriate graft weight is important in liver transplant to provide better graft regeneration and avoid graft failure due to small-for-size syndrome [13, 14].

Multi-slice CTA was used for evaluating liver vascular anatomy; CT was also a useful technique for estimating right-lobe graft volume and right posterior sector graft volume $[11,13,15]$.

Replication of hepatocytes generally starts within 1 day after a major resection [14]. The initiation and synchronization of replication in different types of hepatic cells depend on the extent of the resection, tissue damage, or both. Low-grade tissue damage or a relatively small resection (removal of less than $30 \%$ of the liver) substantially reduces the replication rate, which also appears to be less synchronized than after a large resection (removal of $70 \%$ of the liver) [16-18].

The human body responds to partial hepatectomy not by regenerating lost segments but by inducing hyperplasia in the liver remnant $[16,17,20]$. A study using serial MRI measurements showed that donor and recipient mass increased by $144 \%$ and $99 \%$, respectively by 2 months. 
Regeneration appears to be greatest in the first week. Kamel and colleagues used CT scans to assess liver regeneration in recipients and donors. They found an increase in donor and recipient liver size of $(42 \% \pm 26 \%)$ vs. $(86 \% \pm 11 \%),(67 \% \pm$ $41 \%)$ vs. $(120 \% \pm 27 \%)$, and $(74 \% \pm 46 \%)$ vs. $(75 \% \pm 37 \%)$ at 1,2 , and 6 months, respectively. Of note, the overall the rate of growth was greater in recipients than donors $[12,21$, 22].

In a study done by Kwon et al., to evaluate the extent of liver regeneration and recovery of liver function, Serial CT scan was performed preoperatively, at postoperative day (POD) \#7 and POD \#30. The remained liver volume after harvest increased to $144.6 \%$ at POD \#7, and $181.4 \%$ at POD $\# 30$, which was $88.5 \%$ of preoperative total liver volume [10]. In another report by Marcos he found that donor liver mass increased by $101 \%, 110 \%, 115 \%$, and $144 \%$ at 7,14 , 30 , and 60 days after resection, respectively [6].

According to the literature, the recovery of donor liver volume was still incomplete at 1 year post donation, accounting for $85 \%$ of the total preoperative volume; however, liver function had almost returned to normal [19].
Overall liver regeneration was $83.3 \pm 9.0 \%$ of the total liver volume (TLV) by 1 year in a study done by Pomfret, and there was no effect of age, body mass index (BMI), operative time, estimated blood loss, postoperative complications, or perioperative liver function tests on liver regeneration the latter continued throughout the first postoperative year. Only one donor achieved complete liver regeneration during this time period in this series; however, all donors had maintained normal liver function without long-term complications. Longer follow-up was recommended to determine whether donors ever achieved original TLV [11, 23, 24].

In a more recent study, donor outcome and liver regeneration in 13 males and 14 females were evaluated. Donor RLV was $40.8 \pm 6.6 \%$ of original TLV at surgery, 79.8 $\pm 12.0 \%$ by 6 months, and $97.2 \pm 10.8 \%$ by 12 months. At 3 months the liver of the older donors ( $>$ or $=50$ years) had grown significantly more slowly than in younger donors $(70.4 \pm 9.2 \%$ vs. $79.3 \pm 9.6 \%, \mathrm{P}=0.0391)$ and authors concluded that residual liver regenerated to its preoperative size by 1 year. However, meticulous care should be taken in donors with liver steatosis and aged donor [25, 26, 27].
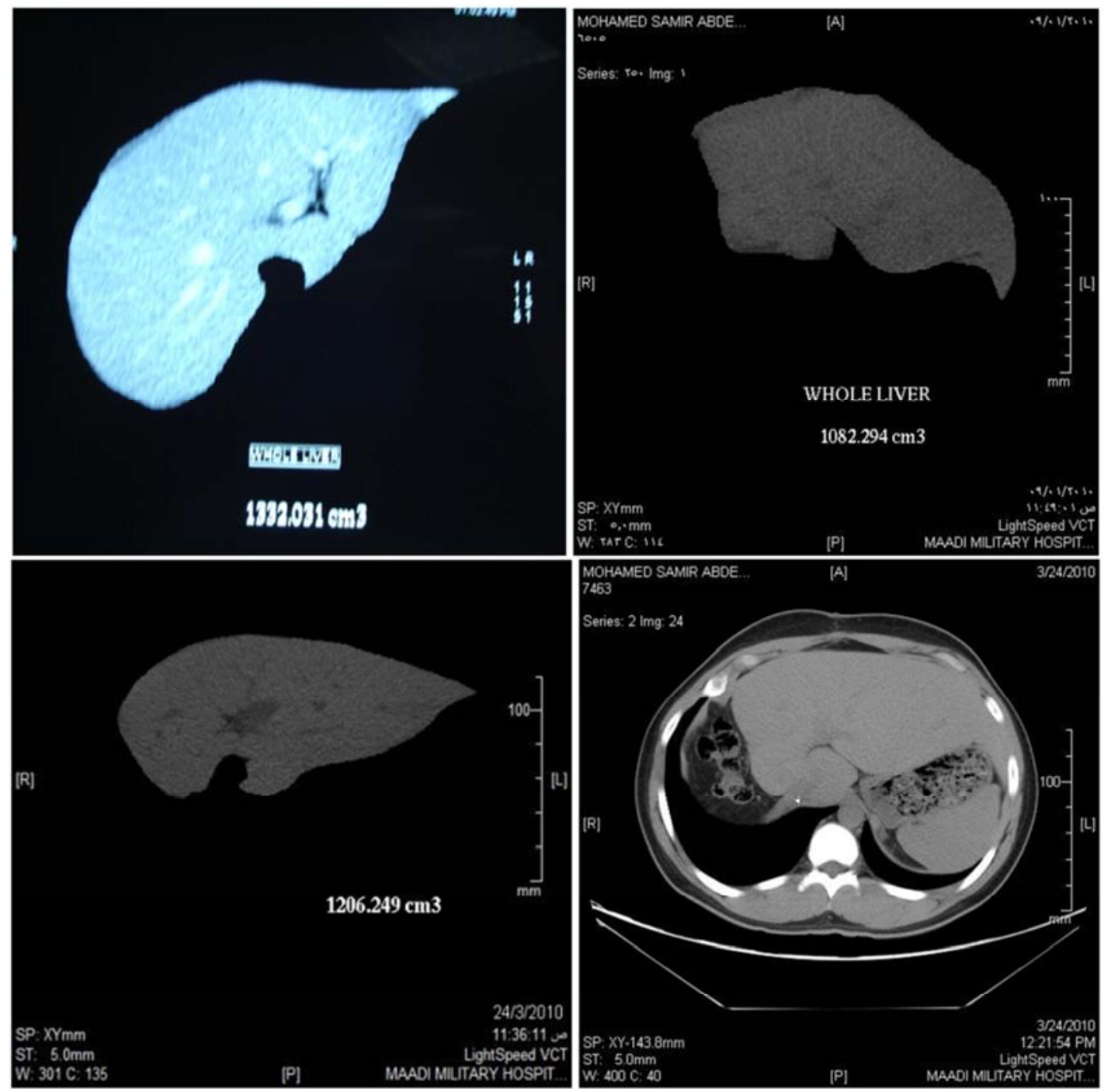

Figure 4. Liver regeneration in right lobe graft, $19 y$ rs male donor. A. Whole liver preoperative $1332 \mathrm{~cm}^{3}$. B. One month, whole liver $81.2 \%$ of TLV. C. 3 months, whole liver $91.7 \%$ of TLV. D. 6 months, whole liver $99.9 \%$ of TLV. 
In this study the regeneration of the remnant liver was significantly different with sex of the donors, age of the donors, type of the graft, size of the remnant liver and the remnant liver / donor weight ratio, and not affected by steatosis of the graft, BMI, operative time, estimated blood loss, postoperative complications, or perioperative liver function tests on liver regeneration. The remnant liver regenerated was more in right lobe graft donors than in right posterior graft donors; the overall liver regeneration was $95.2 \%$ of total liver volume by 6 months in donors of right lobe graft and nearly reached the total liver volume in donors of right posterior sector graft by 3 months.

The volume of the right lobe was bigger in female donors than male donors; we harvested $56.4 \%$ of TLV in female donors and $52.1 \%$ of TLV in male donors that means the remnant liver size in female was less than in males. The remnant liver of female group showed rapid regeneration than male group especially in POD\#7, we found this to be curious, knowing that estrogen induced liver regeneration [11].

Regarding the impact of age on donor prognosis, it has been reported that the length of hospitalization of liver donors aged $>50$ years was longer compared with that of young donors; furthermore, the ability of postoperative protein synthesis is decreased, the duration of cholestasis is prolonged, TB levels increase significantly, and the incidence rate of postoperative complications is higher among older patients. And for recipients receiving an elderly donor liver, there is a significant increase in the risk of postoperative microvascular thrombosis [28, 29, 30]. In our study all of our donors were less than $40 \mathrm{yrs}$ and so no effect of age was found on the rate of regeneration. We found also that the remnant liver of teenagers group showed significant slow regeneration until POD \#7, we couldn't explain the reason of this result. The liver regeneration of this group had no difference to other group in POD\#30, POD\#90 and POD\#180.

Remnant liver also regenerated more rapidly and persisted significantly until POD\#180 in the donors who had a remnant liver volume less than $40 \%$ of TLV. And in donors who had remained liver / donor weight ratio from 0.6 to 0.8 . Our result showed more rapid regeneration of liver in those who had a small remnant liver volume especially early after resection, and the remnant liver volume of $35 \%$ of TLV would be enough to safe recovery of liver function.

The effect of hemodynamic changes in the portal vein on the initiation and promotion of liver regeneration after hepatectomy has been extensively studied in human and animal models. Any factor that is capable of influencing hepatic vascular resistance can possibly modify portal blood flow. Several animal studies have demonstrated that portal vein flow increases immediately after hepatectomy, possibly due to factors such as the induction of urokinase plasminogen activator gene expression and the activation of hepatocyte growth factor [20. 31]. The increased portal flow in the donors who got a less amount of remnant liver and donors who had less remained liver / donor weight ratio might influence more rapid liver regeneration. We checked portal flow increased velocity and volume by duplex US liver at the time interval of CT volumetry [32].

In our program we didn't use donors with fatty liver more than $10 \%$ of steatosis. As a result, we could not find any differences of liver regeneration and function recovery between the donors group with different degree of steatosis.

\section{Conclusion}

The donor liver regenerated up to $95.3 \%$ of preoperative volume at 6 months postoperative with full recovery of liver function at POD \#7. Right lobe donors suffered more complications and need more meticulous operative and postoperative care than right posterior sector graft donors.

The regeneration of the remnant liver was significantly different with sex of the donors, age of the donors, type of the graft, size of the remnant liver and the remnant liver / donor weight ratio, and there was no effect of steatosis of the graft, anatomical vascular and biliary variations, BMI, operative time, estimated blood loss, postoperative complications, or perioperative liver function tests on liver regeneration.

Liver regeneration after donor hepatectomy is affected mainly by how small remnant liver volume, and how much portal flow or portal hypertension to the remnant liver and subsequent compliance of the remnant liver.

\section{References}

[1] Kim WR, Lake JR, Smith JM, Skeans MA, Schladt DP, and Kasiske BL. OPTN/SRTR 2015 Annual data report: Liver. Am J Transplant 2017; S174. S251.

[2] Trotter J: Living donor liver transplantation: is the hype over? $\mathrm{J}$ of hepato. 2005; 42 (1): 20-25.

[3] Olthoff KM, Emond JC, Shearon TH, Everson G, Baker TB, Fisher RA, Freise CE, Gillespie BW and Everhart JE. Liver regeneration after living donor transplantation: Adult-to-adult living donor liver transplantation cohort study. Liver Transpl. 2015; 21: 79-88.

[4] Gruttadauria S, Parikh V, Pagano D, Tuzzolino F, Cintorino D, Miraglia R, et al: Early regeneration of the remnant liver volume after right hepatectomy for living donation: a multiple regression analysis. Liver Transpl. 2012; 18: 907-913.

[5] Chae MS, Moon KU, Chung HS, Park CS, Lee J, Choi JH, et al. Serum interleukin-6 and tumor necrosis-.factor are associated with early graft regeneration after living donor liver transplantation. PLoS One. 2018; 13: e0195262. https://doi.org/10.1371/journal.pone.0195262. PMID: 29649247.

[6] Marcos A, Fisher R, Ham J, Shiffman M, Sanyal A, Luketic $\mathrm{V}$, et al. Liver regeneration and function in donor and recipient after right lobe adult to adult living donor liver transplantation. Transpl. 2000; 69 (7): 1375-1379. 
[7] Botha J, Langnas A, Campos B, Grant W, Freise C, Ascher N, et al. Left lobe adult-to-adult living donor liver transplantation: small grafts and hemiportocaval shunts in the prevention of small for size syndrome. Liver Transpl. 2010; 16: 649-657.

[8] Chen H, Chen C, Huang T, Chen T, Tsang L, Ou H, et al. Regeneration rate of left liver grafts in adult living donor liver transplant. Transplant Proc. 2010; 42: 699-700.

[9] Zhang W, Tan Y, Shen S, Jiang L, Yan L, Yang J, Li B, Wen T, Zeng $\mathrm{Y}$, Wang $\mathrm{W}$ and $\mathrm{Xu} \mathrm{M}$. Adult to adult right lobe living donor liver transplantation: Does biological relationship matter? Medicine (Baltimore). 2017; 96: e4139.

[10] Kwon K, Kim Y, Kim S, Kim K, Lee W, Choi J. Postoperative liver regeneration and complication in live liver donor after partial hepatectomy for living donor liver transplantation. Yonsei medical j. 2003; 44 (6): 1069-1077.

[11] Pomfret E, Pomposelli J, Gordon F, Erbay N, Price L, Lewis $\mathrm{W}$, et al. Liver regeneration and surgical outcome in donors of right - lobe liver grafts. Transpl. 2003; 76 (1): 5-10.

[12] Kamel I, Erbay N, Warmbrand G, Kruskal J, Pomfret E, Raptopoulos V. Liver regeneration after living adult right lobe transplantation. Abdo imag. 2003; 28 (1): 0053-0057.

[13] Wen T, Chen Z, Yan L, Li B, Zeng Y, Zhao J, et al. Measures for increasing the safety of donors in living donor liver transplantation using right lobe grafts. Hepatobiliary Pancreat Dis Int. 2007; 6 (6): 590-595.

[14] Clavien P, Petrowsky H, DeOliveira M, Graf R. Strategies for safer liver surgery and partial liver transplantation. New England J. Med. 2007; 356 (15): 1545-1559.

[15] Broelsch C, Malagó M, Testa G, Gamazo C. Living donor liver transplantation in adults: outcome in Europe. Liver Transpl. 2000; 6 (6B): s64-s65.

[16] Olthoff K. Molecular pathways of regeneration and repair after liver transplantation. World j. surg. 2000; 26 (7): 831837.

[17] Fausto N. Liver regeneration: from laboratory to clinic. Liver transpl. 2001; 7 (10): 835-844.

[18] Taub R. Liver regeneration: from myth to mechanism. Nature Reviews Molecular Cell Biology. 2004; 5 (10): 836-847.

[19] Duclos J, Bhangui P, Salloum C, Andreani P, Saliba F, Ichai P, Elmaleh A, Castaing D and Azoulay D. Ad integrum functional and volumetric recovery in right lobe living donors: Is it really complete 1 year after donor hepatectomy? Am J Transplant. 2016; 16: 143.156.

[20] Eguchi S, Yanaga K, Sugiyama N, Okudaira S, Furui J, Kanematsu T. Relationship between portal venous flow and liver regeneration in patients after living donor right-lobe liver transplantation. Liver transpl. 2003; 9 (6): 547-551.

[21] Chen H, Chen C, Huang T, Chen T, Tsang L, Ou H, et al. Regeneration rate of left liver grafts in adult living donor liver transplant. In Transplantation proc. 2010; 42 (3): 699-700.

[22] Cheng Y, Huang T, Chen T, Tsang L, Ou H, Yu C, et al. Liver graft regeneration in right lobe adult living donor liver transplantation. American J. Transpl. 2009; 9 (6): 1382-1388.

[23] Grewal H, Thistlewaite Jr J, Loss G, Fisher J, Cronin D, Siegel C, et al. Complications in 100 living-liver donors. Annals of surg. 1998; 228 (2): 214.

[24] Schiano T, Kim - Schluger L, Gondolesi G, Miller C. Adult living donor liver transplantation: the hepatologist's perspective. Hepato. 2001; 33 (1): 3-9.

[25] Yokoi H, Isaji S, Yamagiwa K, Tabata M, Sakurai H, Usui M, et al. Donor outcome and liver regeneration after right lobe graft donation. Transplant international. 2005; 18 (8): 915922.

[26] Gruttadauria S, Parikh V, Pagano D, Tuzzolino F, Cintorino D, Miraglia R, et al. Early regeneration of the remnant liver volume after right hepatectomy for living donation: a multiple regression analysis. Liver Transpl. 2012; 18: 907-913.

[27] Klink T, Simon P, Knopp C, Ittrich H, Fischer L, Adam G, et al. Liver remnant regeneration in donors after living donor liver transplantation: long-term follow-up using CT and MR imaging. RoFo. 2014; 186: 598-605.

[28] Imamura H, Hidaka M, Soyama A, Kitasato A, Adachi T, Ono S, Natsuda K, Hara T, Kugiyama T, Baimakhanov Z, et al. A donor age based and graft volume based analysis for living donor liver transplantation in elderly recipients. Transplant Direct 2017; 3: e168.

[29] Kubota T, Hata K, Sozu T, Ueda Y, Hirao H, Okamura Y. donor age on recipient survival in adult to adult living donor liver transplantation. Ann Surg 2018: 267: 1126-1133.

[30] Tanemura A, Mizuno S, Wada H, Yamada T, Nobori T, Isaji S. Donor age affects liver regeneration during early period in the graft liver and late period in the remnant liver after living donor liver transplantation. World J Surg. 2012; 36: 11021111.

[31] Hou C-T, Chen Y-L, Lin C-C, Chou C-T, Lin K-H, Lin P-Y, et al. Portal venous velocity affects liver regeneration after right lobe living donor hepatectomy. PLoS ONE. 2018; 13 (9): e0204163.

[32] Kwon YJ, Lee KG, Choi D. Clinical implications of advances in liver regeneration. Clin Mol Hepatol. 2015; 21: $7 \pm 13$. https://doi.org/10.3350/cmh.2015.21.1.7. PMID: 25834796. 